\title{
SUMS OF ULTRAFILTERS
}

\author{
BY ZDENĚKK FROLfK
}

Communicated by E. Hewitt, June 6, 1966

The main result, an estimate of the cardinality of the set of all ultrafilters producing a given type of ultrafilter (see definition 1.4 and Theorem $C$ in 1.4), is illustrated by a proof of nonhomogeneity of $\beta N-N$ (see 2.1) without using the continuum hypothesis, and by an exhibition of the following two examples.

THEOREM A. For each positive integer $n$ there exists a space $X$ such that $X^{n}$ is countably compact but $X^{n+1}$ is not.

Theorem B. There exists a space $Y$ such that each finite product $Y^{n}$ is countably compact but $Y^{\text {soo }}$ is not.

By a space we mean a separated uniformizable topological space; and $Z^{m}$ stands for the product of any constant family $\{Z \mid a \in A\}$ such that the cardinal of $A$ is $m$.

In our examples the spaces $X^{n+1}$ in A and $Y{ }^{*}$ in B are not pseudocompact. An exhibition of A and B with countably compact replaced by pseudocompact is done in [3]; it does not require Theorem $C$. Trivial examples of spaces with properties in A and B do not seem to be available.

Observe the proof of $\mathrm{A}$ and $\mathrm{B}$ reduces to the following.

THEOREM $\mathrm{A}^{\prime}$. For each positive integer $n$ there exist spaces $X(1), \cdots$, $X(n+1)$ such that the product of any family $\left\{X\left(k_{j}\right) \mid i=1, \cdots, n\right\}$ is countably compact but the product $\{X(j) \mid j \leqq n+1\}$ is not countably compact.

Theorem $\mathrm{B}^{\prime}$. There exists a sequence $\{Y(j)\}$ of spaces such that the product of any finite subfamily is countably compact but the product of $\{Y(j)\}$ is not.

Indeed, for an $X$ in A take the sum of spaces $X(j)$ with properties in $\mathrm{A}^{\prime}$. For $Y$ in B take a one-point countable-compactification of the sum of a sequence $\{Y(j)\}$ with properties in $\mathrm{B}^{\prime}$; then the product of $\{(j) \times Y(j)\}$ is a closed subspace of $Y$.

REMARK. In addition, we shall exhibit $\{Y(j)\}$ such that the product of any proper subfamily (e.g. $\{Y(j) \mid j \geqq 2\}$ ) is countably compact. On the other hand there exists a sequence $\{Y(j)\}$ such that the product of a subfamily is countably compact if and only if the subfamily is finite. 
In what follows $N$ denotes the set and the discrete space of natural numbers, $\beta N$ the Stone-Cech compactification of $N$ such that $N \subset \beta N$ and the points of $\beta N-N$ are free ultrafilters on $N, N^{*}=\beta N-N, P$ the set of all permutations of $N, f^{*}$ with $f$ in $P$ the continuous extension of $f$ to a mapping (homeomorphism) of $\beta N$ onto itself, and $P^{*}$ the set of all $f^{*}$ with $f$ in $P$.

1. Types and production of types. Recall that the cardinal of $\beta N$ is $\exp \exp \boldsymbol{\aleph}_{0}$, the cardinal of every dense set in $N^{*}$ is at least $\exp \boldsymbol{\aleph}_{0}$, and any discrete countable subset of $\beta N$ is normally embedded in $\beta N$.

1.1. Let $T$ be a set and $\tau$ be a mapping of $N^{*}=\beta N-N$ onto $T$ such that $\tau x=\tau y$ if and only if $p^{*} x=y$ for some $p$ in $P$. The elements of $T$ are called the types of ultrafilters on $N$, and if $t=\tau x$ then $t$ is called the type of $x$ and $x$ is said to be of type $t$. The set $\tau^{-1}[(t)]$ of all $x \in N^{*}$ of type $t$ is of cardinal exp $\boldsymbol{\aleph}_{0}$ because the cardinal of $P$ is $\exp \aleph_{0}$ and $\tau^{-1}[(t)]$ is clearly dense in $N^{*}$. It follows that the cardinal of $T$ is $\exp \exp \boldsymbol{\aleph}_{0}$.

If $M$ is any countable infinite set and $f: M \rightarrow N$ is a bijective mapping then the type of any free ultrafilter $x$ on $M$ is defined to be the type of the "image" of $x$ under $f$. Clearly the definition does not depend on $f$.

1.2. If $X$ is any collection of ultrafilters on a set $M$ and if $y$ is an ultrafilter on $X$ then the sum of $X$, with respect to $y$ designated by $\sum_{y} X$, is defined to be the collection $z$ of all sets of the form $U\left\{M_{x} \mid x \in Y\right\}$ where $Y \in y$ and $M_{x} \in x$ for each $x$ in $Y$. It is easy to show that $z$ is actually an ultrafilter on $M$. A collection $X$ of ultrafilters is called to be discrete if there exists a disjoint family $\left\{M_{x} \mid x \in X\right\}$ with $M_{x} \in x$. In a natural way we apply those definitions to a one-toone family $\left\{x_{a}\right\}$ of ultrafilters and an ultrafilter on the index set.

Now let $\left\{x_{n}\right\}$ and $\left\{x_{n}^{\prime}\right\}$ be two discrete sequences of ultrafilters on a countable set $M$ such that $\tau x_{n}=\tau x_{n}{ }^{\prime}$ for each $n$. Then the sums $\sum_{y}\left\{x_{n}\right\}$ and $\sum_{y}\left\{x_{n}^{\prime}\right\}$ are of the same type for any ultrafilter $y$ on $N$, and so we may introduce the following definition.

1.3. Definition. If $\left\{t_{n}\right\}$ is any sequence of types and $y$ is any ultrafilter on $N$ then the sum $t$ of $\left\{t_{n}\right\}$ with respect to $y$ is designated by $\sum_{y}\left\{t_{n}\right\}$ and defined to be the type of any $\sum_{\nu}\left\{x_{n}\right\}$ with $\left\{x_{n}\right\}$ a discrete sequence of ultrafilters such that $\tau x_{n}=t_{n}$. It is clear that then any $x$ of type $t$ is of the form $\sum_{\nu}\left\{x_{n}\right\}$.

Now we are prepared to introduce the main concept-the producing relation.

1.4. Definition. The producing relation $\phi$ on $T$ is defined to be the set of all pairs $\langle u, v\rangle$ such that $v=\sum_{v}\left\{t_{n}\right\}$ for some $y$ of type $u$ 
and some sequence $\left\{t_{n}\right\}$ in $T$. Thus, the domain of $\phi$ is $T$, its range is contained in $T$ and $\phi[(t)]=E\left\{\sum_{y}\left\{t_{n}\right\} \mid y \in t\right\}$. The symbol $\langle u, v\rangle \in \phi$ will of ten be read either " $u$ produces $v$ " or " $v$ is produced by $u$." The main result reads as follows.

Theorem C. Any type is produced by at most exp $\aleph_{0}$ types, and any type produces $\exp \exp \aleph_{0}$ types, i.e. $\operatorname{card} \phi^{-1}[(t)] \leqq \exp \aleph_{0}, \operatorname{card} \phi[(t)]$ $=\exp \exp \aleph_{0}$ for any type $t$.

The proof will be given in 1.7 and 1.9 below after we develop a topological interpretation of the relation $\phi$.

1.5. It is easy to see that a countable $X \subset N^{*}$ is a discrete subset of the topological space $\beta N$ if and only if $X$ is discrete in the sense of 1.2 , and that $\mathrm{cl} X$ is homeomorphic to $\beta N$ if $X$ is infinite. Thus given a $z$ in $\mathrm{cl} X-X$, the traces of neighborhoods of $z$ on $X$ form an ultrafilter $z_{X}$ on $X$ whose type will be denoted by $\tau_{X} z$ and called the type of $z$ relative to $X$. Clearly $z=\sum_{z_{X}} X$ and so $\left\langle\tau_{X} z, t z\right\rangle \in \phi$. If $y$ is any free ultrafilter on $X$ then $z=\sum_{y} X$ belongs to $\mathrm{cl} X-X$ and $y=z_{X}$.

Now let $\left\{t_{n}\right\}$ be any sequence of types and $t$ be a type. Choose a discrete sequence $\left\{x_{n}\right\}$ of representatives (that means $\tau x_{n}=t_{n}$ ), and consider the set of all $x_{n}$. The set of all $\sum_{y}\left\{t_{n}\right\}, \tau y=t$, coincides with the set $E\left\{\tau z \mid z \in \operatorname{cl} X-X, \tau_{X} z=t\right\}$. So $\phi[(t)]=E\{\tau z \mid z \in \mathrm{cl} X-X$, $\tau_{X} z=t$ for some discrete countable $\left.X \subset N^{*}\right\}$. In what follows we shall use that topological interpretation without any reference.

1.6. Let $\left\{M_{n}\right\}$ be a countable decomposition of $N$ and let $x_{n}$, $y_{n} \in \mathrm{cl} M_{n}-M_{n}$. If $x_{n} \neq y_{n}$ for each $n$ then $\operatorname{cl} E\left\{x_{n}\right\} \cap \operatorname{cl} E\left\{y_{n}\right\}=\varnothing$ and conversely. The proof is evident.

1.7. The second statement of Theorem $\mathrm{C}$ follows immediately from 6. Indeed taking any decomposition $\left\{M_{n}\right\}$ of $N$ with all $M_{n}$ infinite, we have card $\mathrm{cl} M_{n}-M_{n}=\exp \exp \aleph_{0}$ for each $n$ and therefore we get $\exp \exp \aleph_{0}$ disjoints sets $\operatorname{cl} X=\operatorname{cl} E\left\{x_{n}\right\}$ each containing at least one point $y$ with $\tau_{X} y=t$, which is of type in $\phi[(t)]$. Since card $\tau^{-1}[(t)]$ $=\exp \aleph_{0}$, the result follows.

To prove the first statement of Theorem $\mathrm{C}$ we need the following lemma.

1.8. Let $y \in \beta N-N$. There exists a set $x$, $\operatorname{card} x \leqq \exp \aleph_{0}$, of discrete countable subsets $X$ of $\beta N-N$ such that if $Y$ is any discrete countable subset of $\beta N-N$, and if $y \in \mathrm{cl} Y-Y$, then $Y \supset X$ for some $X \in \mathcal{x}$.

Proof. For each countable decomposition $\left\{M_{n}\right\}$ of $N$ choose an $x_{n} \in \mathrm{cl} M_{n}-M_{n}$ such that $y \in \mathrm{cl} X-X$, if possible, and take all $X^{\prime} \subset X$ with $y \in \operatorname{cl} X^{\prime}-X$. The set $x$ of all $X^{\prime},\left\{M_{n}\right\}$ variable, has 
required properties by 1.6 . The cardinal of $\mathcal{X}$ is at most $\exp \boldsymbol{\aleph}_{0} \exp \boldsymbol{\aleph}_{0}$ $=\exp \aleph_{0}$.

1.9. To prove the first statement of Theorem $C$ it now suffices to combine 1.7 with 1.5 and the following simple observation: If $X \subset Y$, $y \in \operatorname{cl} X-Y$ and $Y$ is a discrete subset of $\beta N-N$, then $\tau_{X} y=\tau_{Y} y$, i.e. $\tau_{X}$ is a restriction of $\tau_{Y}$.

THEOREM $\mathrm{C}^{\prime}$. Let $\phi_{\infty}=\bigcup\left\{\phi^{k} \mid k \in N, k \neq 0\right\}, \phi_{\infty}^{-1}=\bigcup\left\{\left(\phi^{-1}\right)^{k} \mid k \in N\right.$, $k \neq 0\}$, where $\rho^{k}=\rho \circ \cdots \circ \rho$ (k-times). Then $\phi_{\infty}^{-1}=\left(\phi_{\infty}\right)^{-1}$, and $\operatorname{card} \phi_{\infty}[(t)]=\exp \exp \aleph_{0}$, card $\phi_{\infty}^{-1}[(t)] \leqq \exp \boldsymbol{\aleph}_{0}$ for any $t$ in $T$.

2. Applications. A space is called homogeneous if any point can be mapped on to any point by an autohomeomorphism. W. Rudin proved in [5] that the space $N^{*}$ is not homogeneous by proving the existence of the so called $P$-points. His proof of the existence of $P$-points heavily depends on the continuum hypothesis. Theorem $C$ enables us to prove the nonhomogeneity of $N^{*}$ without the continuum hypothesis.

2.1. Proof of nonhomogeneity of $N^{*}$. For each $x$ in $N^{*}$ denoted by $T_{x}$ the set of all relative types of $x$; i.e. $T_{x}=\phi^{-1}[\tau x]$. If $l x=y$ for some autohomeomorphism $l$ of $N^{*}$, then clearly $T_{x}=T_{y}$. Since the sets $T_{x}$ are of the cardinals at most $\exp \boldsymbol{\aleph}_{0}$, card $T=\exp \exp \aleph_{0}$ and $\left\{T_{x} \mid x \in N^{*}\right\}$ is a covering of $T$, the result follows.

REMARK. It should be remarked that we have proved the existence of $\exp \exp \boldsymbol{\aleph}_{0}$ equivalence classes. Those equivalence classes define "free types" of free ultrafilters. Relative free types are defined similarly.

It remains to prove Theorems $\mathrm{A}^{\prime}$ and $\mathrm{B}^{\prime}$.

2.2. Lemma. There exists a disjoint transfinite family $\left\{T_{\alpha} \mid \alpha<\omega_{1}\right\}$ of subsets of $T$ and a family $\left\{t_{\alpha} \mid \alpha<\omega_{1}\right\}, t_{\alpha} \in T$, such that, denoting by $X_{\alpha}$ the set of all points of $\beta N$ of types in $T_{\alpha}$, each countable discrete subset $X$ of $U\left\{X_{\beta} \mid \beta<\alpha\right\}$ has a cluster point in $X_{\alpha}$ of type $t_{\alpha}$ with respect to $X$.

THEOREM D. For any set $A$ of countable ordinals let $P_{A}$ $=N \cup \cup\left\{X_{\alpha} \mid \alpha \in A\right\}$ be a subspace of $\beta N$. If $\left\{A_{b} \mid b \in B\right\}$ is a countable family of sets of countable ordinals, then the product $P=\prod\left\{P_{A_{b}} \mid b \in B\right\}$ is countably compact if $\cap\left\{A_{\beta}\right\}$ is unbounded, and it is not countably compact if that intersection is empty.

First we prove Theorem $\mathrm{D}$, then Theorems $\mathrm{A}^{\prime}, \mathrm{B}^{\prime}$, and finally the main step, the Lemma.

2.3. Proof of Theorem D. If the intersection is empty then the "diagonal" is a closed infinite discrete subspace of the product. For 
the converse, assume that the intersection is an unbounded set $A$, and let $\{z(n)\}$ be a sequence in $P$. Denoting by $\pi_{b}$ the projection from $P$ onto $P_{A_{b}}$ we can choose a subsequence $\{y(n)\}$ such that each sequence $\left\{\pi_{b} y(n)\right\}$ is either eventually constant or eventually one-toone. Choose an $\alpha \in A$ so that each $\pi_{b} y(n)$ belongs to $U\left\{X_{\beta} \mid \beta<\alpha\right\}$. Choose any point $y \in \beta N$ of type $t_{\alpha}$ and consider the point $z=\left\{z_{b}\right\}$ of $P$ defined as follows: if $\left\{\pi_{b} y(k)\right\}$ is eventually constant, then $z_{b}$ is this constant; otherwise $z_{b}$ is the image of $y$ under the mapping $\left\{n \rightarrow \pi_{b} y(n)\right\}: N \rightarrow N$. It can be proved that $z$ is a cluster point of $\{y(n)\}$, see [3; the proof of $E]$, and so of $\{z(n)\}$.

2.4. Proof of Theorem $\mathrm{A}^{\prime}$. For $0 \leqq k \leqq n$ let $A_{k}$ be the class of countable ordinals which are not congruent to $k$ modulo $n+1$. Of course $\bigcap\left\{A_{k} \mid k \leqq n\right\}=\varnothing$ and the intersection of any proper subfamily of $\left\{A_{k} \mid k \leqq n\right\}$ is unbounded.

2.5. Proof of Theorem $\mathrm{B}^{\prime}$ is similar. For each $k \in N$ let $A_{k}$ be the set of all ordinals which are not congruent to $k$ modulo $\omega_{0}$.

It remains to prove Theorem $\mathrm{D}$. The following simple consequence of Theorem $\mathrm{C}^{\prime}$ will be needed.

2.6. If $T^{\prime} \subset T$ is of cardinal at most $\exp \aleph_{0}$ and if $T_{1} \subset T$ is of cardinal $\exp \exp \boldsymbol{\aleph}_{0}$, then $T^{\prime} \cap \phi^{\infty}[(t)]=\varnothing$ for $\exp \exp \boldsymbol{\aleph}_{0}$ of $t \in T_{1}$. Indeed, by Theorem $\mathrm{C}^{\prime}$ each set $\phi_{\infty}^{-1}[(t)]$ is of cardinal at most $\exp \boldsymbol{\aleph}_{0}$.

2.7. Proof of Lemma. We shall prove the existence of $\left\{T_{\alpha}\right\}$ and $\left\{t_{\alpha}\right\}$ with the following additional properties:

(a) card $T_{\alpha} \leqq \exp \aleph_{0}$;

(b) $T_{\alpha}, \alpha>0$, consists precisely of the types of points of $\beta N$ whose types with respect to some discrete subset of $U\left\{X_{\beta} \mid \beta<\alpha\right\}$ is $t_{\alpha}$.

(c) $\phi\left[\left(t_{\alpha}\right)\right] \cap\left(U\left\{T_{\beta} \mid \beta<\alpha\right\}\right)=\varnothing$.

Starting with any $t=t_{0}, T_{0}=\left(t_{0}\right)$ the induction goes by 2.6.

\section{REFERENCES}

1. E. Cech, Topological spaces, Publ. H. of ČSAV, Praha, 1965.

2. Z. Frolik, The topological product of countably compact spaces, Czech. Math. J. 10 (1960), 329-338.

3. - On two problems of W. W. Comfort, (to appear).

4. J. Novák, On the topological product of two countably compact spaces, Fund. Math. 40 (1953), 106-112.

5. W. Rudin, Homogeneity problems in the theory of Čech compactifications, Duke Math. J. 23 (1956), 409-419, 633.

6. H. Terasaka, On the cartesian product of compact spaces, Osaka Math. J. 4 (1952), 11-15.

Case Institute of Technology 